\title{
Mysterious Looks of Typhoid Fever: A New Experience
}

\author{
Deepankar Kumar Basak ${ }^{1}$, Richmond Ronald Gomes ${ }^{2}$, Md. Ashraf Uddin Ahmed ${ }^{3}$, ABM Sarwar-E-Alam
}

\begin{abstract}
Enteric fever (typhoid or paratyphoid) is a common infectious disease worldwide, especially in developing countries like Bangladesh. Typhoid fever caused by the gram negative bacterium Salmonella typhi that may have a wide spectrum of clinical presentations. We report a case of a 17 year old previously healthy college boy who presented with fever, abdominal pain and shortness of breath later on who was eventually diagnosed as having typhoid fever complicated by acute pancreatitis, hepatitis and severe pulmonary hypertension. Enteric fever presenting as acute pancreatitis and pulmonary hypertension is a rare entity and must be considered in endemic areas like Bangladesh, in patients presenting with fever and concomitant severe abdominal pain and shortness of breath, as any delay in timely institution of treatment can prove fatal. There are very few case reports in the world which have depicted this unusual association.
\end{abstract}

Key words: Acute Pancreatitis, Hepatitis, Enteric Fever, Typhoid, Abdominal pain, Fever, Shortness of Breath, Pulmonary Hypertension

\section{Introduction}

Gastrointestinal complications of enteric fever are intestinal hemorrhage and perforation, acute pancreatitis, hepatic abscess, acute cholecystitis, splenic rupture and hepatitis ${ }^{1}$. Initial gastrointestinal presentation as pancreatitis is an extremely rare manifestation of enteric fever ${ }^{2}$. This atypical presentation has to be borne in mind in dealing with a case of fever with abdominal pain in an endemic country like Bangladesh especially if additional suggestions are present. Pulmonary complications of enteric fever are hemoptysis, pleural effusion, suppurative pneumonia, lung abscess, empyema, bronchopleural fistula ${ }^{3,4}$. So far, in literature search, we have not found a case report of pulmonary hypertension associated with typhoid fever.

\section{Case Report}

A 17 year old college student hailing from Dhaka, admitted into Square Hospital Limited, Dhaka, Bangladesh through emergency room with the complaints of fever for 8 days, dry cough, anorexia, abdominal fullness and loose motion for several times per day for the same duration. Fever was initially low grade, intermittent, associated with chills and rigor and subsided spontaneously. Later fever became high

1. Dr. Deepankar Kumar Basak, MBBS, FCPS (Medicine), Specialist-Gastroenterology, Square Hospitals Ltd

2. Dr. Richmond Ronald Gomes, MBBS, FCPS (Medicine), Assistant Professor, Internal Medicine, Ad-din Women's Medical College \& Hospital, Dhaka

3. Dr. Md. Ashraf Uddin Ahmed, MBBS, FCPS (Medicine), Specialist-Internal Medicine, Square Hospitals Ltd

4. Dr. ABM Sarwar-E-Alam, MBBS, FCPS (Medicine), Consultant, Internal Medicine, Square Hospitals Limited

Corresponding Author:

Dr. Deepankar Kumar Basak

MBBS, FCPS (Medicine)

Specialist-Gastroenterology

Square Hospital Ltd, Dhaka

E-mail: deepankarbasak@gmail.com grade, intermittent (max recorded temperature $104^{\circ} \mathrm{F}$ ) with chills and rigor and subsided only by taking anti pyretic. Along with fever he also had dry cough with scanty mucoid sputum. But there was no $\mathrm{H} / \mathrm{O}$ chest pain, haemoptysis, shortness of breath. He was also having anorexia, abdominal fullness and passage of loose watery stool several times per day since then. But there was no blood with stool \& abdominal pain. $\mathrm{He}$ had no $\mathrm{H} / \mathrm{O}$ headache, altered consciousness, convulsion, throat pain, dysuria, weight loss, any rash or bleeding from any site or any joint pain. He neither had any significant travel history nor had any contact with active TB patient. He was treated locally by GP with twice daily $500 \mathrm{mg}$ Cefuroxime for 7 days prior admission as blood C/S from outside showed growth of Salmonella typhi.

On examination he was ill looking, co operative, febrile with average body built, tongue was coated. There was no skin or nail changes, bony tenderness, peripheral lymphadenopathy or thyromegaly. Pulse-72/min, BP-110/70 $\mathrm{mmHg}$, Temp- $101^{\circ} \mathrm{F}$. Abdomen was distended with no visible vein, peristalsis, and pulsation. Liver was enlarged about $5 \mathrm{~cm}$ from right costal margin along right MCL, firm, non tender, sharp margin, Upper border of liver dullness was present in right $5^{\text {th }}$ ICS in right MCL. There was no hepatic bruit or rub. Spleen was also enlarged, about $3 \mathrm{~cm}$ along its long axis, non tender. There was no splenic rub. There was ascites, no other intra abdominal mass, para aortic adenopathy. Bowel sound was normal. On auscultation of lung breath sound was diminished on basal region. Cardiovascular, neurological and locomotor system examination revealed no abnormalities.

Initial lab reports showed CBC- Hb- $12.4 \mathrm{~g} / \mathrm{dl}$, TC-4.60K/uL with $\mathrm{N}-75.5 \%$, TPC $72000 \mathrm{~K} / \mathrm{uL}$, ESR $9 \mathrm{~mm} / 1 \mathrm{st}$ hour, S. Bilirubin 0.9mg/dl, AST 759u/L, ALT 408u/L, CRP 298.6, Tripple antigen was non conclusive. Urine R/E- albumin ++, no RBC, cast, pus cell. USG of W/A revealed hepatosplenomegaly with mild ascites and $\mathrm{B} / \mathrm{L}$ mild pleural effusion. Renal function was normal. Stool routine examination was normal. After sending the blood for $\mathrm{C} / \mathrm{S}$, Inj. Ceftriaxone was started thinking of typhoid fever. Repeat blood C/S showed growth of Salmonella typhi sensitive to 
Ceftriaxone. With treatment his fever started responding. Frequency of loose motion was decreasing, but he developed upper abdominal pain. Repeat clinical examination revealed increasing ascites \& B/L pleural effusion with left parasternal lift, loud P2 with systolic murmur over left lower parasternal area. There was epigastric and right hypochondriac tenderness. He became pale \& again developed high fever with progressive $\mathrm{SOB}$. SaO 2 was $92 \%$ with $3 \mathrm{~L} / \mathrm{min}$ oxygen. There was no bipedal edema. Repeat $\mathrm{CBC}$ revealed $\mathrm{Hb}-9.90$ $\mathrm{g} / \mathrm{dl}$, TC $5.30 \mathrm{~K} / \mathrm{uL}, \mathrm{N}-52.3 \%$, TPC $62000 \mathrm{~K} / \mathrm{uL}$, CRP 68.2. All viral markers (HAV, HEV, HCV, HBV) were negative, LDH 3954U/L, Lipase 1180U/L, S. Bilirubin 1.3mg/dl, ALT 367u/l, AST 673u/1, ALP 329u/l, S. Albumin 2.1g/dl, S. Reticulocyte $0.46 \%$. Anti dengue antibody was negative. PT, aPTT were normal. PBF was non conclusive. Repeat ultrasonography of abdomen revealed prominent periportal lymph node, increasing ascites and B/L pleural effusion. Direct coombs test was negative. Pleural fluid tap revealed transudative fluid. Echocardiography showed no pericardial effusion with PASP $50 \mathrm{~mm}$ of $\mathrm{Hg}$ with moderate MR and mild TR. CT abdomen with contrast revealed prominent ileo-colic and para aortic node, largest one being measures $12 \mathrm{~mm}$, Pleural fluid for $\mathrm{C} / \mathrm{S}$ and MTB-PCR, MT, ANA all were negative.
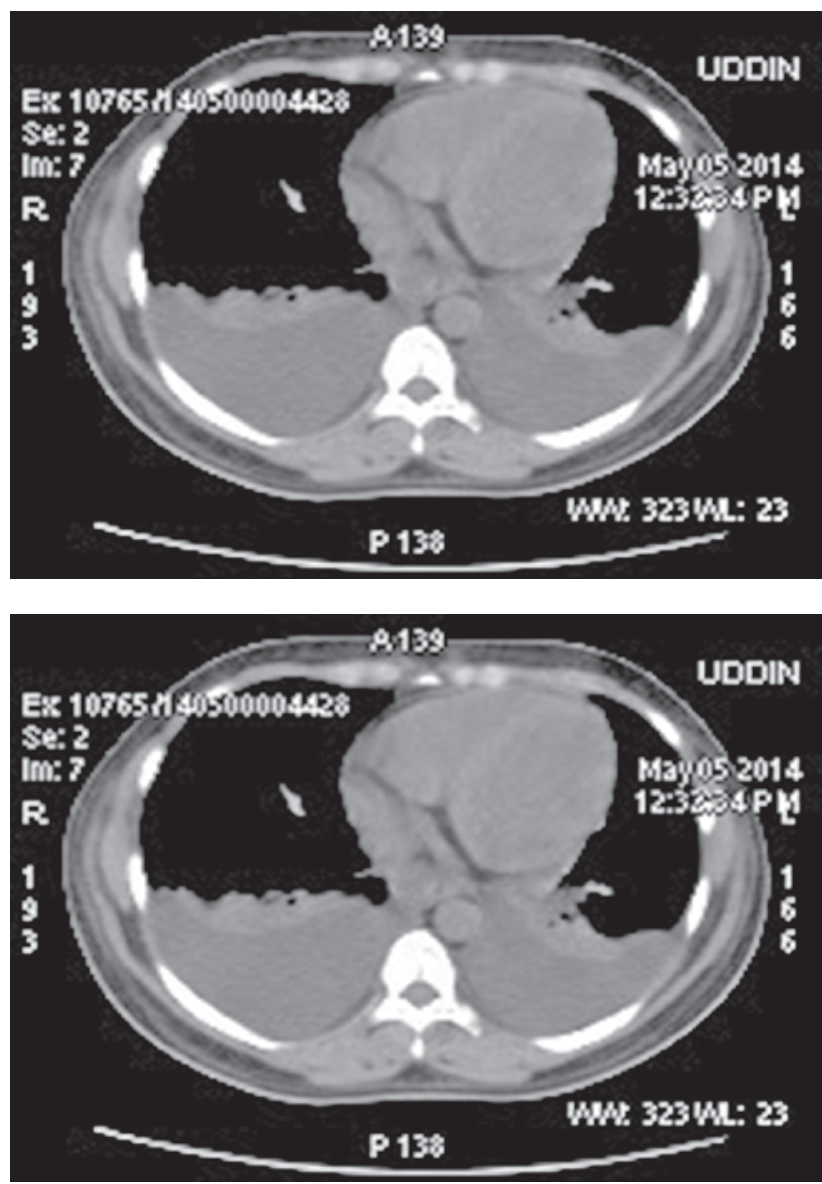

Fig 1 \& 2 - Pleural effusion

Patient was still dyspnoeic with $\mathrm{SaO} 293 \%$ with $2 \mathrm{~L} / \mathrm{min} \mathrm{O} 2$, febrile with temperature $102^{\circ} \mathrm{F}$. There is a plan for intra abdominal node biopsy or bone marrow study to rule out Non
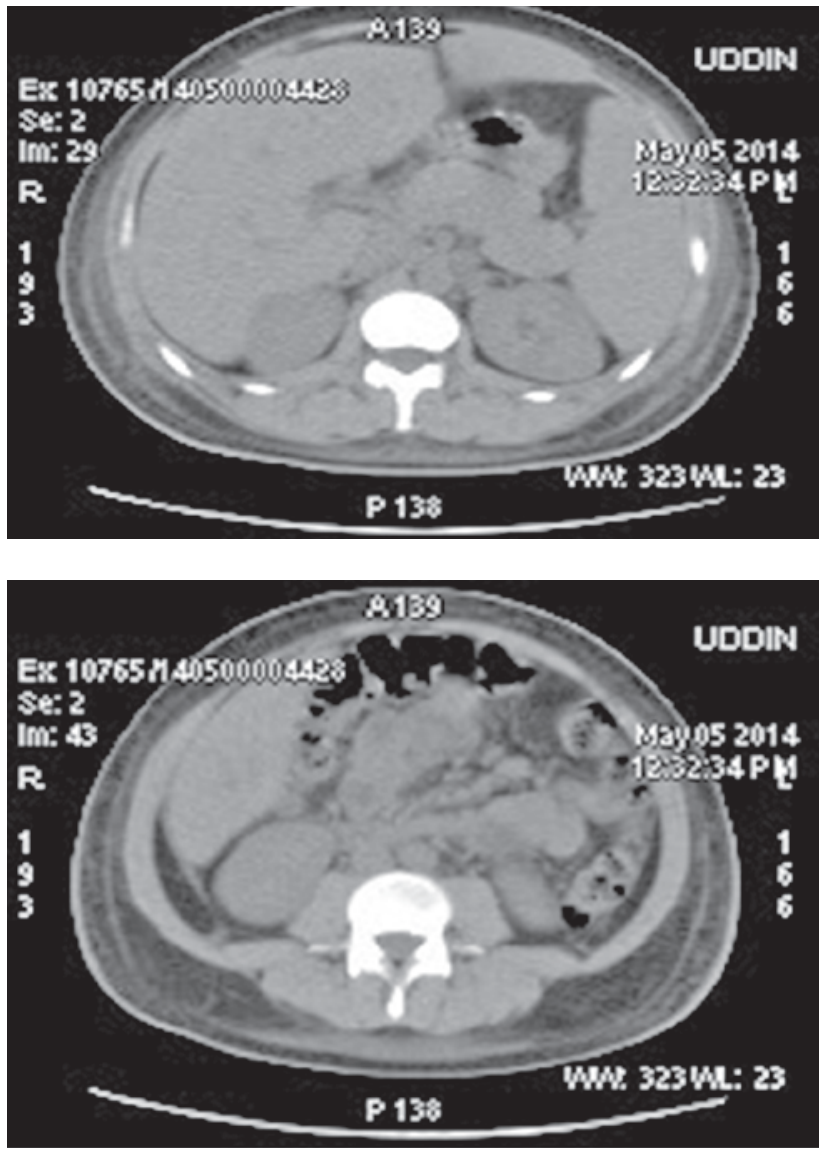

Fig 3 \& 4 - Hepatosplenomegaly and ileo-colic and para aortic nodes

Hodgkins Lymphoma. But later the antibiotic was changed to Injection Meropenem. He was treated for acute pancreatitis with nothing per oral, analgesics. He was monitored closely for development of cor pulmonale. With conservative treatment (Inj. Meropenem was given as 1 gram thrice daily dose for 10 days) he had shown significant clinical improvement as evidenced by subsidence of fever, shortness of breath and abdominal complaints. Follow up CT abdomen revealed decreasing size of previous lymph nodes, liver and spleen. During discharge his Complete blood count, Liver function tests and serum Lipase all were improved. PASP became $20 \mathrm{~mm}$ of $\mathrm{Hg}$ with no MR or TR on follow up echocardiography.

\section{Discussion}

Patients with enteric fever may present with abdominal pain and the causes for abdominal pain in a patient with enteric fever are intestinal haemorrhage and perforation, acute cholecystitis, typhoid hepatitis, hepatic abscess, splenic rupture and acute pancreatitis ${ }^{1,2}$. Enteric fever masquerading as acute pancreatitis is a rare entity ${ }^{5}$. The abnormalities of pancreas that are associated with typhoid fever may include simple biochemical abnormalities such as hyperamylasemia to acute pancreatitis, pancreatic abscess, chronic pancreatitis, pancreatic pseudocyst requiring surgery ${ }^{6,7}$. Retrospective studies have reported a frequency of hyperamylasemia of 
$50 \%$ and a frequency of clinical pancreatitis ranging from $28 \%$ to $62 \%$ in patients with Salmonella infection ${ }^{8}$. Few case reports have highlighted the association of acute pancreatitis and other pancreatic complications with Salmonella infections. Two cases of acute pancreatitis by Salmonella typhi were reported by Koshi'. One of these cases required distal pancreatectomy while the other required only drainage of pseudocyst. The first case of pancreatic abscess due to Salmonella typhimurium was reported by Strand and Sanders. ${ }^{9}$. The notable feature was that the case had associated gallstones and chronic cholecystitis. The index patient here had normal gall bladder and biliary tract. Khan et al reported a case of enteric fever complicated by acute pancreatitis, acute renal failure and rhabdomyolysis ${ }^{10}$. In our case, no renal failure was noted and no evidence of rhabdomyolysis was present. Localized Salmonella infection of the pancreas is usually the result of Salmonella bacteraemia caused by Salmonella choleraesuis but may also occur after gastroenteritis by $\mathrm{S}$. typhimurium and enteric fever by Salmonella typhi ${ }^{11}$. Once pancreatitis develops, it may form a pancreatic abscess. Pancreatic pseudocyst may occasionally be infected by Salmonella typhi ${ }^{2}$. Salmonella typhi is known to localize in injured or damaged tissue or insites of malignancy ${ }^{5}$. Kune and Coster reported a case of pancreatic abscess due to Salmonella typhi who had gallstones; the organism was isolated from the necrotic pancreatic tissue ${ }^{12}$. Mechanism of pancreatitis in enteric fever is not exactly known. Typhoid pancreatitis could represent the effect of the direct pancreatic localisation of bacteria which could happen by haematogenous route, lymphatic route and transmural migration, via the biliary duct system and from the duodenum via the main pancreatic duct. This may occur, especially in patients with predisposing conditions to biliary stasis, such as cholelithiasis, choledocholithiasis, and biliary duct abnormalities ${ }^{13}$, but our patient had no such predisposing conditions. The mechanisms may also be toxin induced or immune mediated pancreatitis ${ }^{14,15}$. Isolated hyperamylasemia and hyperlipasemia without evidence of pancreatic involvement could also be the result of a reduced excretion due to either impaired renal or liver function which is common in Salmonella infections. Concomitant hepatitis (as in our case) and renal failure may contribute to cause abnormally elevated levels of amylase and lipase than what would be expected by isolated pancreatitis alone ${ }^{16}$. Gnadinger MP et al suggested that the excess of hyperamylasemia seen in Salmonella infections may be due to intestinal inflammation leading to abnormally increased absorption of macromolecules like amylase; he demonstrated an increased intestinal permeability for oral 51Cr- EDTA in two patients with elevated amylase serum levels in the course of entero-invasive salmonellosis. However, other studies have failed to reproduce this finding ${ }^{17}$. Treatment includes aggressive management of both typhoid fever and acute pancreatitis ${ }^{5}$. Treatment with anti-secretory drugs, by reducing the gastric killing of ingested organisms, has been shown to represent a risk factor for severity and mortality, so its administration is strongly discouraged ${ }^{18}$.

\section{Conclusion}

This case highlights the importance of keeping in mind the possibility of development of acute pancreatitis and pulmonary hypertension in a case of enteric fever as appropriate diagnosis and treatment might lead to complete resolution of the potentially fatal condition. Salmonella infection must be considered in cases of non-alcoholic or nonlithiasic pancreatitis.

\section{References}

1. Koshi G. Uncommon manifestations of salmonella infections. Indian Journal of Medical Research. 1976;64(2):314-321.

2. Bhan MK, Bahl R, Bhatnagar S. Typhoid and paratyphoid fever. Lancet. 2005;366:749-762.

3. Hovette P, Camara P, Petrognani R, Donzel C. 1998;58(4):403-7

4. Bouvier B, Metman EH, Girard JJ, Groussin P. 1978 Sep $8-15 ; 54(21-24): 755-7$

5. Hermans P, Gerard M, van Laethem Y, de Wit S, Clumeck N. Pancreatic disturbances and typhoid fever. Scandinavian Journal of Infectious Diseases. 1991;23(2):201-205.

6. Renner F, Nimeth C, Demmelbauer N. High frequency of concomitant pancreatitis in Salmonella enteritis. Lancet. 1991;337(8757):1611.

7. Kadappu KK, Rao PV, Srinivas N, Shastry BA. Pancreatitis in enteric fever. Indian Journal of Gastroenterology. 2002;21(1):32-33.

8. Renner F, Nimeth C, Demmelbauer N. High frequency of concomitant pancreatitis in

Salmonella enteritis. Lancet 1991;337:1611.

9. Strand CL, Sanders SL. Salmonella typhimurium pancreatic abscess - report of a case. Am Surg. 1978, 44:174-176.

10. Khan FH, Al-Ani A, AliHA. Typhoid rhabdomyolysis with acute renal failure and acute pancreatitis: a case report and review of the literature.Int J Infect Dis. 2009;13(5):e282e285.

11. Cohen JI, Bartlett, JA, Correy GR. Extraintestinal manifestations of salmonella infection. Medicine. 1987;66:349-388.

12. Kune, GA, Coster D. Typhoid pancreatic abscess. Med J Aust. 1972;1:417-418.

13. Hearne SE, Whigham TE, Brady CE. Pancreatitis and typhoid fever. Am J Med. 1989;86:471- 473.

14. Arendt T. Bile-induced acute pancreatitis in cats. Roles of bile, bacteria, and pancreatic duct pressure. Dig Dis Sci. 1993;38(1):39-44.

15. Schmid SW, Uhl W, Friess H, Malfertheiner P, Büchler MW. The role of infection in acute pancreatitis. Gut. 1999;45(2):311-316.

16. Pezzilli R, Andreone P, Morselli-Labate AM, Sama C, Billi P, Cursaro C, et al. Serum pancreatic enzyme concentrations in chronic viral liver diseases. Dig Dis Sci.1999;44:350-355.

17. Gnadinger MP, Eigenmann F, Bekier A, Galeazzi RL. Pseudopancreatitis in entero-invasive salmonellosis. Schweiz Med Wochenschr. 1993;123:1482-1486.

18. Neal K, Briji SO, Slack RC, Hawkey CJ, Logan RF. Recent treatment with $\mathrm{H} 2$ antagonists and antibiotics and gastric surgery as risk factors for Salmonella infection. BMJ. 1994;308:176. 